\title{
On the Maximal Colorings of Complete Graphs Without Some Small Properly Colored Subgraphs
}

\author{
Chunqiu Fang $^{1,2,4} \cdot$ Ervin Györi $^{2}$ (D) Jimeng Xiao ${ }^{2,3,5}$
}

Received: 14 December 2019/Revised: 10 May 2021 / Accepted: 4 June 2021 /

Published online: 15 June 2021

(C) The Author(s) 2021

\begin{abstract}
Let $\operatorname{pr}\left(K_{n}, G\right)$ be the maximum number of colors in an edge-coloring of $K_{n}$ with no properly colored copy of $G$. For a family $\mathcal{F}$ of graphs, let ex $(n, \mathcal{F})$ be the maximum number of edges in a graph $G$ on $n$ vertices which does not contain any graphs in $\mathcal{F}$ as subgraphs. In this paper, we show that $\operatorname{pr}\left(K_{n}, G\right)-\operatorname{ex}\left(n, \mathcal{G}^{\prime}\right)=o\left(n^{2}\right)$, where $\mathcal{G}^{\prime}=\{G-M: M$ is a matching of $G\}$. Furthermore, we determine the value of $\operatorname{pr}\left(K_{n}, P_{l}\right)$ for sufficiently large $n$ and the exact value of $\operatorname{pr}\left(K_{n}, G\right)$, where $G$ is $C_{5}, C_{6}$ and $K_{4}^{-}$, respectively. Also, we give an upper bound and a lower bound of $\operatorname{pr}\left(K_{n}, K_{2,3}\right)$.
\end{abstract}

Keywords Properly colored subgraphs · Turán numbers · Anti-Ramsey numbers

C. Fang: supported in part by CSC(No. 201806210164). E. Györi: supported in part by the National Research, Development and Innovation Office NKFIH, Grants K116769, K117879 and K126853.

J. Xiao: supported in part by CSC (No. 201706290171).

Ervin Győri

gyori.ervin@ renyi.mta.hu

Chunqiu Fang

fcq15@tsinghua.org.cn

Jimeng Xiao

xiaojimeng@mail.nwpu.edu.cn

1 Department of Mathematical Sciences, Tsinghua University, Beijing 100084, China

2 Alfréd Rényi Institute of Mathematics, ELKH, Reáltanoda u.13-15, Budapest 1053, Hungary

3 Department of Applied Mathematics, Northwestern Polytechnical University, Xi'an, China

4 Yau Mathematical Sciences Center, Tsinghua University, Beijing 10084, China

5 Xi'an-Budapest Joint Research Center for Combinatorics, Northwestern Polytechnical University, Xi'an, China 


\section{Introduction}

We call a subgraph of an edge-colored graph rainbow, if all of its edges have different colors. While a subgraph is called properly colored (also can be called locally rainbow), if any two adjacent edges receive different colors. The antiRamsey number of a graph $G$ in a complete graph $K_{n}$, denoted by $\operatorname{ar}\left(K_{n}, G\right)$, is the maximum number of colors in an edge-coloring of $K_{n}$ with no rainbow copy of $G$. Namely, $\operatorname{ar}\left(K_{n}, G\right)+1$ is the minimum number $k$ of colors such that any $k$-edgecoloring of $K_{n}$ contains a rainbow copy of $G$. In this paper, we let $\operatorname{pr}\left(K_{n}, G\right)$ be the maximum number of colors in an edge-coloring of $K_{n}$ with no properly colored copy of $G$. Namely, $\operatorname{pr}\left(K_{n}, G\right)+1$ is the minimum number $k$ of colors such that any $k$ edge-coloring of $K_{n}$ contains a properly colored copy of $G$.

Given a family $\mathcal{F}$ of graphs, we call a graph $G$ an $\mathcal{F}$-free graph, if $G$ contains no graph in $\mathcal{F}$ as a subgraph. The Turán number $\operatorname{ex}(n, \mathcal{F})$ is the maximum number of edges in a graph $G$ on $n$ vertices which is $\mathcal{F}$-free. Such a graph $G$ is called an extremal graph, and the set of extremal graphs is denoted by $\operatorname{EX}(n, \mathcal{F})$. The celebrated result of Erdös-Stone-Simonovits Theorem [7,9] states that for any $\mathcal{F}$ we have

$$
\operatorname{ex}(n, \mathcal{F})=\left(\frac{p-1}{2 p}+o(1)\right) n^{2},
$$

where $p=\Psi(\mathcal{F})=\min \{\chi(F): F \in \mathcal{F}\}-1$, is the subchromatic number.

The anti-Ramsey number was introduced by Erdős, Simonovits and Sós in [8]. There they showed that $\operatorname{ar}\left(K_{n}, G\right) \geq \operatorname{ex}(n, \mathcal{G})+1$, where $\mathcal{G}=\{G-e: e \in E(G)\}$ and by (1.1), they showed that $\operatorname{ar}\left(K_{n}, G\right)=\left(\frac{d-1}{2 d}+o(1)\right) n^{2}$, where $d=\Psi(\mathcal{G})$. This determined $\operatorname{ar}\left(K_{n}, G\right)$ asymptotically when $\Psi(\mathcal{G}) \geq 2$. In the case $\Psi(\mathcal{G})=1$, the situation is more complex. Already the cases when $G$ is a tree or a cycle are nontrival. For a path $P_{k}$ on $k$ vertices, Simonovits and Sós [20] proved $\operatorname{ar}\left(K_{n}, P_{2 t+3+\epsilon}\right)=t n-\left(\begin{array}{c}t+1 \\ 2\end{array}\right)+1+\epsilon$, for large $n$, where $\epsilon=0$ or 1. Jiang [11] showed $\operatorname{ar}\left(K_{n}, K_{1, p}\right)=\left\lfloor\frac{n(p-2)}{2}\right\rfloor+\left\lfloor\frac{n}{n-p+2}\right\rfloor$ or possibly this value plus one if certain conditions hold. For a general tree $T$ of $k$ edges, Jiang and West [12] proved $\frac{n}{2}\left\lfloor\frac{k-2}{2}\right\rfloor+O(1) \leq \operatorname{ar}\left(K_{n}, T\right) \leq \operatorname{ex}(n, T)$ for $n \geq 2 k$ and conjectured that $\operatorname{ar}\left(K_{n}, T\right) \leq \frac{k-2}{2} n+O(1)$. For cycles, Erdős, Simonovits and Sós [8] conjectured that for every fixed $k \geq 3, \operatorname{ar}\left(K_{n}, C_{k}\right)=\left(\frac{k-2}{2}+\frac{1}{k-1}\right) n+O(1)$, and proved that for $k=3$. Alon [1] proved this conjecture for $k=4$ and gave some upper bounds for $k \geq 5$. Finally, Montellano-Ballesteros and Neumann-Lara [18] completely proved this conjecture, that is, for $n \geq k \geq 3$ and $n \equiv r_{k}(\bmod (\mathrm{k}-1))$, where $0 \leq r_{k} \leq k-2$,

$$
\operatorname{ar}\left(K_{n}, C_{k}\right)=\left\lfloor\frac{n}{k-1}\right\rfloor\left(\begin{array}{c}
k-1 \\
2
\end{array}\right)+\left(\begin{array}{c}
r_{k} \\
2
\end{array}\right)+\left\lceil\frac{n}{k-1}\right\rceil-1 .
$$

For cliques, Erdős, Simonovits and Sós [8] showed $\operatorname{ar}\left(K_{n}, K_{p+1}\right)=\operatorname{ex}\left(n, K_{p}\right)+1$ for $p \geq 3$ and sufficiently large $n$. Montellano-Ballesteros and Neumann-Lara [17] and independently Schiermeyer [19] showed that $\operatorname{ar}\left(K_{n}, K_{p+1}\right)=\operatorname{ex}\left(n, K_{p}\right)+1$ holds for 
every $n \geq p \geq 3$. For complete bipartite graphs, Axenovich and Jiang [2] showed that $\operatorname{ar}\left(K_{n}, K_{2, t}\right)=\operatorname{ex}\left(n, K_{2, t-1}\right)+O(n)$, where $t \geq 2$. Krop and York [13] showed that $\operatorname{ar}\left(K_{n}, K_{s, t}\right)=\operatorname{ex}\left(n, K_{s, t-1}\right)+O(n)$, where $t \geq s \geq 2$. Also, there are many other results about anti-Ramsey numbers. We mention the excellent survey by Fujita, Magnant and Ozeki [10] for more conclusions on this topic.

The maximum number of colors in an edge-colored complete graph without some properly colored subgraphs was first studied by Manoussakis, Spyratos, Tuza and Voigt in [15]. For cliques, they [15] obtained the approximate value of $\operatorname{pr}\left(K_{n}, K_{t}\right)$.

Theorem 1 [15] For $t \geq 3$, let $b=\left\lfloor\frac{t-1}{2}\right\rfloor$, we have $\operatorname{pr}\left(K_{n}, K_{t}\right)=\left(\frac{b-1}{2 b}+o(1)\right) n^{2}$.

For paths and cycles, they [15] showed that $\operatorname{pr}\left(K_{n}, P_{n}\right)=\left(\begin{array}{c}n-3 \\ 2\end{array}\right)+1$ for large $n$ and $\operatorname{pr}\left(K_{n}, C_{n}\right)=\left(\begin{array}{c}n-1 \\ 2\end{array}\right)+1$. Also, they gave a conjecture about cycles as follows.

Conjecture 1 [15] Let $n>l \geq 4$. Assume that $K_{n}$ is colored with at least $k$ colors, where

$$
k=\left\{\begin{array}{l}
\frac{1}{2} l(l+1)+n-l+1, \text { if } n<\frac{10 l^{2}-6 l-18}{6(l-3)} \\
\frac{1}{3} l n-\frac{1}{18} l(l+3)+2, \text { if } n \geq \frac{10 l^{2}-6 l-18}{6(l-3)},
\end{array}\right.
$$

then $K_{n}$ admits a properly colored cycle of length $l+1$.

In this paper, we generalize Theorem 1 to an arbitrary graph $G$ which shows that $\operatorname{pr}\left(K_{n}, G\right)$ is related to the Turán number like the anti-Ramsey number.

Theorem 2 Let $G$ be a graph and $\mathcal{G}^{\prime}=\{G-M: M$ is a matching of $G\}$, then $\operatorname{pr}\left(K_{n}, G\right) \geq \operatorname{ex}\left(n, \mathcal{G}^{\prime}\right)+1$ and $\operatorname{pr}\left(K_{n}, G\right)=\left(\frac{d-1}{2 d}+o(1)\right) n^{2}$, where $d=\Psi\left(\mathcal{G}^{\prime}\right)$.

We will prove Theorem 2 in Sect. 2 by the method used in the proof of Theorem 1 in [15]. Theorem 2 determines $\operatorname{pr}\left(K_{n}, G\right)$ asymptotically when $\Psi\left(\mathcal{G}^{\prime}\right) \geq 2$. As the anti-Ramsey number, the case $\Psi\left(\mathcal{G}^{\prime}\right)=1$ is more complex.

In Sect. 3, we will determine $\operatorname{pr}\left(K_{n}, P_{l}\right)$ for large $n$ by proving the following theorem.

Theorem 3 Let $P_{l}$ be a path on $l$ vertices and $l \equiv r_{l}(\bmod 3)$, where $0 \leq r_{l} \leq 2$. For $n \geq 2 l^{3}$, we have

$$
\operatorname{pr}\left(K_{n}, P_{l}\right)=\left(\left\lfloor\frac{l}{3}\right\rfloor-1\right) n-\left(\begin{array}{c}
\left\lfloor\frac{l}{3}\right\rfloor \\
2
\end{array}\right)+1+r_{l} .
$$

For cycles, we slightly improve the lower bound of Conjecture 1 (See Proposition 4). Also, We modify Conjecture 1 as follows. 
Conjecture 2 Let $C_{k}$ be a cycle on $k$ vertices and $(k-1) \equiv r_{k-1}(\bmod 3)$, where $0 \leq r_{k-1} \leq 2$. For $n \geq k$,

$$
\operatorname{pr}\left(K_{n}, C_{k}\right)=\max \left\{\left(\begin{array}{c}
k-1 \\
2
\end{array}\right)+n-k+1,\left\lfloor\frac{k-1}{3}\right\rfloor n-\left(\left\lfloor\frac{k-1}{3}\right\rfloor+1\right)+1+r_{k-1}\right\} .
$$

It is easy to see that $\operatorname{pr}\left(K_{n}, C_{3}\right)=\operatorname{ar}\left(K_{n}, C_{3}\right)=n-1$. Also, by Proposition 4 and (1.2), one can check that for $n \geq 3$,

$$
\begin{gathered}
\operatorname{pr}\left(K_{n}, C_{n}\right)=\operatorname{ar}\left(K_{n}, C_{n}\right)=\left(\begin{array}{c}
n-1 \\
2
\end{array}\right)+1, \\
\operatorname{pr}\left(K_{n+1}, C_{n}\right)=\operatorname{ar}\left(K_{n+1}, C_{n}\right)=\left(\begin{array}{c}
n-1 \\
2
\end{array}\right)+2 .
\end{gathered}
$$

Li, Broersma and Zhang [14], and later Xu, Magnant and Zhang [21] showed that for $n \geq 4, \operatorname{pr}\left(K_{n}, C_{4}\right)=n$. We obtain the exact value of $\operatorname{pr}\left(K_{n}, C_{5}\right)$ and $\operatorname{pr}\left(K_{n}, C_{6}\right)$ in Sect. 4.

Theorem 4 For $n \geq 5, \operatorname{pr}\left(K_{n}, C_{5}\right)=n+2$.

Theorem 5 For $n \geq 6, \operatorname{pr}\left(K_{n}, C_{6}\right)=n+5$.

Let $K_{4}^{-}$be the diamond, the graph obtained from $K_{4}$ by deleting an edge. We obtain the exact value of $\operatorname{pr}\left(K_{n}, K_{4}^{-}\right)$in Sect. 5 .

Theorem 6 For $n \geq 3, \operatorname{pr}\left(K_{n}, K_{4}^{-}\right)=\left\lfloor\frac{3(n-1)}{2}\right\rfloor$.

We also give a lower bound and an upper bound of $\operatorname{pr}\left(K_{n}, K_{2,3}\right)$ in Section 5 .

Theorem 7 For $n \geq 5, \frac{7}{4} n+O(1) \leq \operatorname{pr}\left(K_{n}, K_{2,3}\right) \leq 2 n-1$.

Notations: Let $G$ be a simple undirected graph. For $x \in V(G)$, we denote the neighborhood and the degree of $x$ in $G$ by $N_{G}(x)$ and $d_{G}(x)$, respectively. The maximum degree of $G$ is denoted by $\Delta(G)$. The common neighborhood of $U \subset$ $V(G)$ is the set of vertices in $V(G) \backslash U$ that are adjacent to each vertex of $U$. We will use $G-x$ to denote the graph that arises from $G$ by deleting the vertex $x \in V(G)$. For a vertex set $X \subset V(G), G[X]$ is the subgraph of $G$ induced by $X$ and $G-X$ is the subgraph of $G$ induced by $V(G) \backslash X$. Given a graph $G=(V, E)$, for any (not necessarily disjoint) vertex sets $A, B \subset V$, we let $E_{G}(A, B):=\{u v \in E(G) \mid u \neq v, u \in A, v \in B\}$. We use $\bar{G}$ to denote the complement of $G$. Given two vertex disjoint graphs $G_{1}$ and $G_{2}$, we denote by $G_{1}+G_{2}$ the join of graphs $G_{1}$ and $G_{2}$, that is the graph obtained from $G_{1} \cup G_{2}$ by joining each vertex of $G_{1}$ with each vertex of $G_{2}$.

Given an edge-coloring $c$ of $K_{n}$, we denote the color of an edge $u v$ by $c(u v)$. For any vetex $v \in V(G)$, let $C(v):=\left\{c(v w): w \in V\left(K_{n}\right) \backslash\{v\}\right\}$ and $d_{c}(v):=|C(v)|$. A 
color $a$ is starred (at $x$ ) if all the edges with color $a$ induce a star $K_{1, r}$ (centered at the vertex $x)$. We let $d^{c}(v)=\mid\{a \in C(v): a$ is starred at $v\} \mid$. For a subgraph $H$ of $G$, we denote $C(H)=\{c(u v): u v \in E(H)\}$. A representing subgraph of an edge-colored $K_{n}$ is a spanning subgraph containing exactly one edge of each color. The weak representing subgraph of an edge-colored $K_{n}$ is consisting of all the edges whose color appears only once in $K_{n}$. Note that an edge $x y$ is the unique edge with color $a$ in $K_{n}$ if and only if the color $a$ is stared at both $x$ and $y$. Thus, if $G$ is the weak representing subgraph of an edge-colored $K_{n}$, then we have

$$
|E(G)| \geq \sum_{v \in V\left(K_{n}\right)} d^{c}(v)-\left|C\left(K_{n}\right)\right|
$$

\section{The Proof of Theorem 2}

In this section, we will prove Theorem 2 by a similar argument used in the proof of Theorem 1 in [15].

Theorem 2 Let $G$ be a graph and $\mathcal{G}^{\prime}=\{G-M: M$ is a matching of $G\}$, then $\operatorname{pr}\left(K_{n}, G\right) \geq \operatorname{ex}\left(n, \mathcal{G}^{\prime}\right)+1$ and $\operatorname{pr}\left(K_{n}, G\right)=\left(\frac{d-1}{2 d}+o(1)\right) n^{2}$, where $d=\Psi\left(\mathcal{G}^{\prime}\right)$.

Proof Let $F$ be a graph in $\operatorname{EX}\left(n, \mathcal{G}^{\prime}\right)$. We color the edges of $K_{n}$ as follows. Take a subgraph $F$ of $K_{n}$, and assign distinct colors to all of $E(F)$ and a new color $c_{0}$ to all the remaining edges. Suppose there is a properly colored $G$, then $M=\{e \in$ $E(G), e$ is colored with $\left.c_{0}\right\}$ is a matching of $G$, and $G-M \subset F$. By the definition of $\mathcal{G}^{\prime}$, we have $G-M \in \mathcal{G}^{\prime}$, and this is a contradiction with $F$ being $\mathcal{G}^{\prime}$-free. Thus we have $\operatorname{pr}\left(K_{n}, G\right) \geq \operatorname{ex}\left(n, \mathcal{G}^{\prime}\right)+1=\left(\frac{d-1}{2 d}+o(1)\right) n^{2}$ by (1.1).

Let $G_{0}=G-M_{p}$, where $M_{p}$ is a $p$-matching of $G$ and $\chi\left(G_{0}\right)=d+1$. We prove that for every fixed $\varepsilon>0$, and for $n$ large enough with respect to $n_{0}=|V(G)|$ and $\varepsilon$, there is a properly colored copy of $G$ in any $\left(\frac{d-1}{2 d}+\varepsilon\right) n^{2}$-edge-coloring of $K_{n}$. In a representing subgraph of $K_{n}$ with $\left(\frac{d-1}{2 d}+\varepsilon\right) n^{2}$ edges, for an arbitrarily fixed $s$, and for $n$ sufficiently large, by (1.1), there exists a complete $(d+1)$-partite subgraph $K_{s, s, \ldots, s}$ with $s$ vertices in each class. We take $s=2^{n_{0}+d+1}$.

Denote by $V$ the vertex set of $K_{s, s, \ldots, s}$ and by $V_{1}, V_{2}, \ldots, V_{d+1}$ its vertex classes. We apply the following procedure.

For each $i=1,2, \ldots, d+1$ do sequentially the following:

(1) Select arbitrarily $2^{n_{0}+d+1-i}$ pairwise disjoint pairs $\left\{u_{i j}, v_{i j}\right\}$ in $V_{i}$, $j=1,2, \ldots, 2^{n_{0}+d+1-i}$.

(2) For $j=1,2, \ldots, 2^{n_{0}+d+1-i}$, delete from $K_{s, s, \ldots, s}$ the (at most one) vertex $z \in$ $V \backslash V_{i}$ for which either $c\left(z u_{i j}\right)=c\left(u_{i j} v_{i j}\right)$ or $c\left(z v_{i j}\right)=c\left(u_{i j} v_{i j}\right)$, and if $z$ has already been selected in a previous pair $\left\{u_{i^{\prime} j^{\prime}}, v_{i^{\prime} j^{\prime}}\right\}$, for some $i^{\prime}<i$, then also delete the other member of its pair.

Claim 1 The above procedure can be executed smoothly and there are at least $2^{n_{0}}$ pairs remains undeleted in each $V_{i}$ at the end of the execution. 
The Proof of Claim 1 In the beginning, each $V_{i}$ contains $2^{n_{0}+d+1}$ vertices, $i=1,2, \ldots, d+1$. In the first iteration, $i=1$, we can carry out (1) and (2) easily. Suppose we have carried out up to the $(i-1)$-st iteration. Before executing the $i$-th iteration observe that at most $\sum_{1 \leq j \leq i-1} 2^{n_{0}+d+1-j}=2^{n_{0}+d+1}-2^{n_{0}+d+2-i}$ vertices have been deleted from $V_{i}$. Thus, $V_{i}$ contains at least $2^{n_{0}+d+2-i}$ vertices and it is enough to execute instruction (1) in the $i$ th iteration.

On the other hand, for any $i=1,2, \ldots, d$, from the $(i+1)$-st iteration up to the end, due to instructions of type (2), at most $\sum_{i+1 \leq j \leq d+1} 2^{n_{0}+d+1-j}=2^{n_{0}+d+1-i}-$ $2^{n_{0}}$ pairs in $V_{i}$ have been deleted and thus at least $2^{n_{0}}$ pairs in $V_{i}$ remains undeleted. Note also that $V_{d+1}$ contains $2^{n_{0}}$ pairs of vertices and there is no deletion of pair in $V_{d+1}$.

For $1 \leq i \leq d+1$, let $\left\{x_{i j} y_{i j}: 1 \leq j \leq 2^{n_{0}}\right\}$ be the $2^{n_{0}}$ pairs in $V_{i}$ which remain undeleted and $V_{i}^{\prime}=\left\{x_{i j}, y_{i j}: 1 \leq j \leq 2^{n_{0}}\right\}$. Let $H$ be the graph obtained by adding the edge set $\left\{x_{i j} y_{i j}: 1 \leq i \leq d+1,1 \leq j \leq 2^{n_{0}}\right\}$ to the graph $K_{s, s, \ldots, s}\left[V_{1}^{\prime} \cup \cdots \cup V_{d+1}^{\prime}\right]$. Then $H$ is properly colored by Claim 1 . Since $G_{0}=G-M_{p}$ and $\chi\left(G_{0}\right)=d+1$, we have $H \supset G$. Thus $\operatorname{pr}\left(K_{n}, G\right) \leq\left(\frac{d-1}{2 d}+o(1)\right) n^{2}$.

\section{Paths}

In this section, we study the maximum number of colors in an edge-colored complete graph without properly edge-colored paths, and prove Theorem 3 . Before doing so, we determine $\operatorname{pr}\left(K_{n}, P_{l}\right)$ for some small values of $l$.

\section{Proposition 1}

(a) $\operatorname{pr}\left(K_{n}, P_{3}\right)=1$, for $n \geq 3$.

(b) $\operatorname{pr}\left(K_{n}, P_{4}\right)=2$, for $n \geq 4$.

(c) $\operatorname{pr}\left(K_{n}, P_{5}\right)=3$, for $n \geq 5$.

\section{Proof}

(a) The conclusion holds trivially.

(b) Choose a vertex $v$ of $K_{n}$, color all edges incident to $v$ with color $c_{1}$ and color all the remaining edges with color $c_{2}$. We use two colors and there is no properly colored $P_{4}$. Hence $\operatorname{pr}\left(K_{n}, P_{4}\right) \geq 2$.

For $n \geq 5$, we have $\operatorname{pr}\left(K_{n}, P_{4}\right) \leq \operatorname{ar}\left(K_{n}, P_{4}\right)=2$ (see [3]). For $n=4$, let $V\left(K_{4}\right)=\{u, v, x, y\}$. Given a 3-edge-coloring of $K_{4}$, there exists at least one edge in $E(\{u, v\},\{x, y\})$, we say $u x$, such that $c(u x) \neq c(u v)$ and $c(u x) \neq c(x y)$. Thus vuxy is a properly colored $P_{4}$ and $\operatorname{pr}\left(K_{n}, P_{4}\right) \leq 2$.

(c) Choose two vertices $u$ and $v$ of $K_{n}$, assign one color $c_{1}$ to all edges incident with $u$, one new color $c_{2}$ to all edges incident with $v$ (except the edge $u v$ ) and the other new color $c_{3}$ to all the remaining edges. We use three colors and there is no properly colored $P_{5}$. Hence $\operatorname{pr}\left(K_{n}, P_{5}\right) \geq 3$. 
Let $n \geq 5$. Given a 4-edge-coloring of $K_{n}$, there is always a rainbow $P_{4}=u_{1} u_{2} u_{3} u_{4}$ since $\operatorname{ar}\left(K_{n}, P_{4}\right)=2$ (see [3]). Since $\left|C\left(P_{4}\right)\right|=\left|E\left(P_{4}\right)\right|=3$, there is a color $c_{0} \in C\left(K_{n}\right) \backslash C\left(P_{4}\right)$. Suppose there is no properly colored $P_{5}$ in the 4-edge-coloring of $K_{n}$. Then for all $u \in V\left(K_{n}\right) \backslash V\left(P_{4}\right)$, it must be $c\left(u u_{1}\right)=c\left(u_{1} u_{2}\right)$, $c\left(u u_{4}\right)=c\left(u_{3} u_{4}\right), c\left(u u_{2}\right) \in\left\{c\left(u_{1} u_{2}\right), c\left(u_{2} u_{3}\right)\right\}$ and $c\left(u u_{3}\right) \in\left\{c\left(u_{2} u_{3}\right), c\left(u_{3} u_{4}\right)\right\}$. If $c\left(u_{1} u_{4}\right)=c_{0}$, then $u u_{1} u_{4} u_{3} u_{2}$ is a properly colored $P_{5}$, a contradiction. If $c\left(u_{1} u_{3}\right)=$ $c_{0}$ or $c\left(u_{2} u_{4}\right)=c_{0}$, say $c\left(u_{1} u_{3}\right)=c_{0}$, then $u_{4} u u_{1} u_{3} u_{2}$ is a properly colored $P_{5}$, a contradiction. So we may assume that there are two vertices $x, y \in V\left(K_{n}\right) \backslash V\left(P_{4}\right)$ such that $c(x y)=c_{0}$. In this case, $u_{4} y x u_{2} u_{1}$ or $u_{4} y x u_{2} u_{3}$ is a properly colored $P_{5}$, a contradiction. Hence $\operatorname{pr}\left(K_{n}, P_{5}\right) \leq 3$.

Here, we give the lower bound of $\operatorname{pr}\left(K_{n}, P_{l}\right)$ by the following proposition.

Proposition 2 Let $P_{l}$ be a path on $l$ vertices and $l \equiv r_{l}(\bmod 3)$, where $0 \leq r_{l} \leq 2$. For $n \geq l$, we have

$$
\operatorname{pr}\left(K_{n}, P_{l}\right) \geq \max \left\{\left(\begin{array}{c}
l-3 \\
2
\end{array}\right)+1,\left(\left\lfloor\frac{l}{3}\right\rfloor-1\right) n-\left(\left\lfloor\frac{l}{3}\right\rfloor\right)+1+r_{l}\right\} .
$$

Proof We color the edges of $K_{n}$ as follows. For the first lower bound, we choose a $K_{l-3}$ and color it rainbow, and use one extra color for all the remaining edges. In such way, we use exactly $\left(\begin{array}{c}l-3 \\ 2\end{array}\right)+1$ colors and do not obtain a properly colored $P_{l}$.

For the second lower bound, we partition $K_{n}$ into two graphs $K_{\left\lfloor\frac{l}{3}\right\rfloor-1}+\bar{K}_{n-\left\lfloor\frac{l}{3}\right\rfloor+1}$ and $K_{n-\left\lfloor\frac{l}{3}\right\rfloor+1}$. First we color $K_{\left\lfloor\frac{l}{3}\right\rfloor-1}+\bar{K}_{n-\left\lfloor\left\lfloor\frac{l}{3}\right\rfloor+1\right.}$ rainbow. Then we color $K_{n-\left\lfloor\frac{l}{3}\right\rfloor+1}$ by $\left(1+r_{l}\right)$ new colors without producing a properly colored $P_{3+r_{l}}$ (See the proof of Proposition 3.1). In such way, we use exactly $\left(\left\lfloor\frac{l}{3}\right\rfloor-1\right) n-\left(\left\lfloor\frac{l}{3}\right\rfloor\right)+1+r_{l}$ colors and do not obtain a properly colored $P_{l}$.

The proof of the following proposition is trivial. We will use it to prove Theorem 3.

Proposition 3 Let $P_{l}$ be a path with $l$ vertices, and $l \equiv r_{l}(\bmod 3)$, where $0 \leq r_{l} \leq 2$. If an edge-colored $K_{n}$ contains a rainbow copy of $K_{\left\lfloor\frac{l}{3}\right\rfloor-1,2\left\lfloor\frac{l}{3}\right\rfloor+3}$ but does not contain a properly colored $P_{l}$. We denote by $Q$ the vertices of $K_{n}-K_{\left\lfloor\frac{l}{3}\right\rfloor-1,2\left\lfloor\frac{l}{3}\right\rfloor+3}$, by $X$ the smaller class of $K_{\left\lfloor\frac{l}{3}\right\rfloor-1,2\left\lfloor\frac{l}{3}\right\rfloor+3}$ and by $Y$ the other one. Then $\left|C\left(K_{n}[Y]\right)\right| \leq 1+r_{l}$. Furthermore, we have $\left|C\left(K_{n}[Y]\right) \cup C\left(E_{K_{n}}(Y, Q)\right)\right| \leq 1+r_{l} \quad$ and $\left|C\left(K_{n}[Y \cup Q]\right)\right| \leq 1+r_{l}$. We get the most colors if the colors of all the edges between $X$ and $Y \cup Q$ and all the edges in $X$ are different, they differ from all the other edges and we use exactly $1+r_{l}$ colors in $Y \cup Q$ such that there is no properly colored $P_{3+r_{l}}$ in $Y \cup Q$. Then the number of colors is 


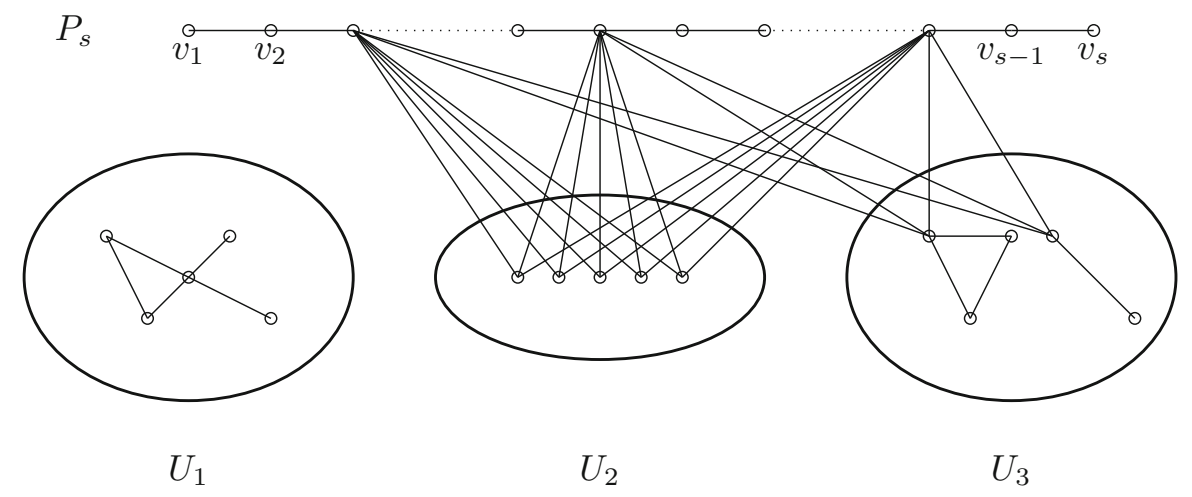

Fig. 1 The structure of graph $G$

$$
\left(\left\lfloor\frac{l}{3}\right\rfloor-1\right) n-\left(\begin{array}{c}
\left\lfloor\frac{l}{3}\right\rfloor \\
2
\end{array}\right)+1+r_{l} .
$$

Now, we will prove Theorem 3, and the idea comes from [20] (Fig. 1).

Theorem 3 Let $P_{l}$ be a path on $l$ vertices and $l \equiv r_{l}(\bmod 3)$, where $0 \leq r_{l} \leq 2$. For $n \geq 2 l^{3}$, we have

$$
\operatorname{pr}\left(K_{n}, P_{l}\right)=\left(\left\lfloor\frac{l}{3}\right\rfloor-1\right) n-\left(\begin{array}{c}
\left\lfloor\frac{l}{3}\right\rfloor \\
2
\end{array}\right)+1+r_{l} .
$$

Proof We just need prove the upper bound for $l \geq 6$. We shall use the following famous results of Erdös and Gallai (see [5]): for $n \geq r \geq 2$,

$$
\begin{gathered}
\operatorname{ex}\left(n, P_{r}\right) \leq \frac{r-2}{2} n, \\
\operatorname{ex}\left(n,\left\{C_{r+1}, C_{r+2}, \ldots\right\}\right) \leq \frac{r(n-1)}{2} .
\end{gathered}
$$

Let $c$ be an edge-coloring of $K_{n}$ using $\operatorname{pr}\left(K_{n}, P_{l}\right)$ colors without producing a properly colored $P_{l}$. Take a longest properly colored path $P_{s}=v_{1} v_{2} \cdots v_{s}$, where $s \leq l-1$. Denote by $G$ the graph obtained by choosing one edge from each remaining color such that the number of edges joining $P_{s}$ to the remaining $n-s$ vertices is as large as possible. We would partition $V(G) \backslash V\left(P_{s}\right)$ into three sets $U_{1}, U_{2}$ and $U_{3}$ as follows:

(a) $\quad U_{1}$ is the vertex set of $V\left(K_{n}\right) \backslash V\left(P_{s}\right)$ not jointed to $P_{s}$ at all: neither by edges nor by paths; 
(b) $U_{2}$ is the set of isolated vertices of $V\left(K_{n}\right) \backslash V\left(P_{s}\right)$ jointed to $P_{s}$ by edges;

(c) $U_{3}=V\left(K_{n}\right) \backslash\left(V\left(P_{s}\right) \cup U_{1} \cup U_{2}\right)$.

Claim 1 For any vertex $u \in U_{1} \cup U_{2} \cup U_{3}$, we have $c\left(u v_{1}\right)=c\left(v_{1} v_{2}\right)$ and $c\left(u v_{s}\right)=c\left(v_{s-1} v_{s}\right)$. Moreover, $E_{G}\left(U_{2} \cup U_{3},\left\{v_{1}, v_{2}, v_{s-1}, v_{s}\right\}\right)=\emptyset$.

Proof of Claim 1 It is obvious that $c\left(u v_{1}\right)=c\left(v_{1} v_{2}\right)$ and $c\left(u v_{s}\right)=c\left(v_{s-1} v_{s}\right)$ for any vertex $u \in U_{1} \cup U_{2} \cup U_{3}$ by the maximality of $P_{s}$, thus we have $E_{G}\left(U_{2} \cup U_{3},\left\{v_{1}, v_{s}\right\}\right)=\emptyset$. Suppose that there is a vertex $u \in U_{2} \cup U_{3}$ such that $u v_{2} \in E(G)$ or $u v_{s-1} \in E(G)$, we say $u v_{2} \in E(G)$. Notice that $c\left(u v_{1}\right)=c\left(v_{1} v_{2}\right) \neq$ $c\left(u v_{2}\right)$ by the definition of $G$, it follows that $v_{1} u v_{2} \cdots v_{s}$ is a properly colored path of order $s+1$, a contradiction to the maximality of $P_{s}$.

Claim $2 G\left[U_{1}\right]$ contains no $P_{\left\lfloor\frac{s}{2}\right\rfloor}$.

Proof of Claim 2 Suppose $P_{\left\lfloor\frac{s}{2}\right\rfloor}=u_{1} u_{2} \ldots u_{\left\lfloor\frac{s}{2}\right\rfloor}$ is a path in $G\left[U_{1}\right]$. By the definition of $G$, the colors of $C\left(G\left[U_{1}\right]\right)$ can not appear in any edges between $U_{1}$ and $V\left(P_{s}\right)$. Thus, $c\left(u_{1} v_{\left\lceil\frac{s}{2}\right\rceil}\right) \neq c\left(u_{1} u_{2}\right), c\left(u_{\left\lfloor\frac{s}{2}\right\rfloor} v_{1}\right) \neq c\left(u_{\left\lfloor\frac{s}{2}\right\rfloor} u_{\left\lfloor\frac{s}{2}\right\rfloor-1}\right)$ and $c\left(u_{\left\lfloor\frac{s}{2}\right\rfloor} v_{s}\right) \neq c\left(u_{\left\lfloor\frac{s}{2}\right\rfloor} u_{\left\lfloor\frac{s}{2}\right\rfloor-1}\right)$. Since $c\left(v_{\left\lceil\frac{s}{2}\right\rceil-1} v_{\left\lceil\frac{s}{2}\right\rceil}\right) \neq c\left(v_{\left\lceil\frac{s}{2}\right\rceil} v_{\left\lceil\frac{s}{2}\right\rceil+1}\right)$, at most one of $c\left(v_{\left\lceil\frac{s}{2}\right\rceil-1} v_{\left\lceil\frac{s}{2}\right\rceil}\right)$ and $c\left(v_{\left\lceil\frac{s}{2}\right\rceil} v_{\left\lceil\frac{s}{2}\right\rceil+1}\right)$ is the same as $c\left(u_{\left\lceil\frac{s+1}{2}\right\rceil} v_{\left\lceil\frac{s}{2}\right\rceil}\right)$. So at least one of $v_{1} v_{2} \ldots v_{\left\lceil\frac{s}{2}\right\rceil} u_{1} u_{2} \ldots u_{\left\lfloor\frac{s}{2}\right\rfloor} v_{s}$ and $v_{s} v_{s-1} \ldots v_{\left\lceil\frac{s}{2}\right\rceil} u_{1} u_{2} \ldots u_{\left\lfloor\frac{s}{2}\right\rfloor} v_{1}$ is a properly colored path of order at least $s+1$, a contradiction to the maximality of $P_{s}$. Hence, $G\left[U_{1}\right]$ contains no $P_{\left[\frac{s}{2}\right\rfloor}$.

By Claim 2 and (3.1), we have

$$
\left|E\left(G\left[U_{1}\right]\right)\right| \leq \frac{1}{2}\left(\left\lfloor\frac{s}{2}\right\rfloor-2\right)\left|U_{1}\right| \leq\left(\frac{1}{2}\left\lfloor\frac{l-1}{2}\right\rfloor-1\right)\left|U_{1}\right| .
$$

Claim 3 For any vertex $u \in U_{2} \cup U_{3}$ and any three consecutive vertices $v_{i}, v_{i+1}, v_{i+2} \in V\left(P_{s}\right)$, we have $\left|E_{G}\left(u,\left\{v_{i}, v_{i+1}, v_{i+2}\right\}\right)\right| \leq 1$.

Proof of Claim 3 Suppose there exist a vertex $u \in U_{2} \cup U_{3}$ and three consecutive vertices $v_{i}, v_{i+1}, v_{i+2} \in V\left(P_{s}\right)$ such that $\left|E_{G}\left(u,\left\{v_{i}, v_{i+1}, v_{i+2}\right\}\right)\right| \geq 2$, that is at least two of $u v_{i}, u v_{i+1}, u v_{i+2}$ are edges of $G$, then whatever $c\left(v v_{i}\right)$ is, at least one of $v_{1} \ldots v_{i} u v_{i+1} v_{i+2} \ldots v_{s}$ and $v_{1} \ldots v_{i} v_{i+1} u v_{i+2} \ldots v_{s}$ is a properly colored path of order $s+1$, a contradiction to the maximality of $P_{s}$.

By Claims 1 and 3, we have $\left|E_{G}\left(u, P_{s}\right)\right| \leq\left\lceil\frac{s-4}{3}\right\rceil \leq\left\lceil\frac{l-5}{3}\right\rceil=\left\lfloor\frac{l}{3}\right\rfloor-1$ for all $u \in U_{2}$. Thus, we have

$$
\left|E_{G}\left(U_{2}, P_{s}\right)\right| \leq\left(\left\lfloor\frac{l}{3}\right\rfloor-1\right)\left|U_{2}\right| .
$$

Let $H$ be any component of $G\left[U_{3}\right]$ and $r$ be the length of the longest cycle in $H$. If $H$ contains no cycles, then we write $r=2$. By (3.2), we have 


$$
|E(H)| \leq \frac{r|V(H)|-r}{2} .
$$

Now we will estimate the number of edges between $V(H)$ and $V\left(P_{s}\right)$ in $G$ by the following two claims.

Claim 4 For any vertex $u \in V(H)$, we have

$$
E_{G}\left(u,\left\{v_{1}, \ldots, v_{2 r+1}, v_{s-2 r}, \ldots, v_{s}\right\}\right)=\emptyset .
$$

Proof of Claim 4 Since $H$ is connected and the length of the longest cycle in $H$ is $r$, we can always find a path $P_{r} \subset H$ starting from $u$ in $H$. Let $P_{r}=u_{1} u_{2} \ldots u_{r}$ be such a path, where $u_{1}=u$. By an argument very similar to the one in Claim 1, we have $E_{G}\left(u,\left\{v_{1}, \ldots, v_{r+1}, v_{s-r}, \ldots, v_{s}\right\}\right)=\emptyset$. By the symmetry, we just need to show that there is no edge between $u$ and $\left\{v_{r+2}, \ldots, v_{2 r+1}\right\}$. If there exists $v_{i} \in\left\{v_{r+2}, \ldots, v_{2 r}\right\}$ such that $u v_{i} \in E(G)$, we have $i \geq r+2 \geq 4$. By the definition of $G$, we have $c\left(u_{r} v_{\left\lfloor\frac{i}{2}\right\rfloor}\right) \neq c\left(u_{r-1} u_{r}\right)$. Since $c\left(v_{\left\lfloor\frac{i}{2}\right\rfloor-1} v_{\left\lfloor\frac{i}{2}\right\rfloor}\right) \neq c\left(v_{\left\lfloor\frac{i}{2}\right\rfloor} v_{\left\lfloor\frac{i}{2}\right\rfloor+1}\right)$, at most one of $c\left(v_{\left\lfloor\frac{i}{2}\right\rfloor-1} v_{\left\lfloor\frac{i}{2}\right\rfloor}\right)$ and $c\left(v_{\left\lfloor\frac{i}{2}\right\rfloor} v_{\left\lfloor\frac{i}{2}\right\rfloor+1}\right)$ is the same as $c\left(u_{r} v_{\left\lfloor\frac{i}{2}\right\rfloor}\right)$. Thus at least one of $v_{1} v_{2} \ldots v_{\left\lfloor\frac{i}{2}\right\rfloor} u_{s} \ldots u_{1} v_{i} v_{i+1} \ldots v_{s}$ and $v_{i-1} v_{i-2} \ldots v_{\left\lfloor\frac{i}{2}\right\rfloor} u_{s} \ldots u_{1} v_{i} v_{i+1} \ldots v_{s}$ is a properly colored path of order at least $s+1$, a contradiction to the maximality of $P_{s}$. If $u v_{2 r+1} \in E(G)$, then we have $c\left(u v_{2 r}\right) \neq c\left(v_{2 r} v_{2 r+1}\right)$, otherwise $v_{1} v_{2} \cdots v_{2 r} u v_{2 r+1} v_{2 r+2} \cdots v_{s}$ is a properly colored path of order $s+1$, a contradiction to the maximality of $P_{s}$. Also, we have $c\left(u v_{2 r}\right) \neq c\left(u u_{2}\right)$. By an argument similar to the above, one can find a properly colored path of order at least $s+1$, a contradiction to the maximality of $P_{s}$.

Claim 5 For any six consecutive vertices $v_{i}, v_{i+1}, v_{i+2}, v_{i+3}, v_{i+4}, v_{i+5} \in V\left(P_{s}\right)$, all edges between $\left\{v_{i}, v_{i+1}, v_{i+2}, v_{i+3}, v_{i+4}, v_{i+5}\right\}$ and $V(H)$ of $G$ induce a star.

Proof of Claim 5 If not, suppose $x v_{i}$ and $y v_{j}$ are two independent edges between $V(H)$ and $\left\{v_{i}, v_{i+1}, v_{i+2}, v_{i+3}, v_{i+4}, v_{i+5}\right\} \quad$ in $G$, where $x, y \in V(H)$ and $j \in\{i+1, i+2, i+3, i+4, i+5\}$. Let $P_{x y}$ be a path of $H$ which connect $x$ and $y$. If $j \in\{i+1, i+2, i+3\}$, then whatever $c\left(x v_{i+1}\right)$ is, at least one of $v_{1} \ldots v_{i} x v_{i+1} \ldots v_{s}$ and $v_{1} \ldots v_{i} v_{i+1} x P_{x y} y v_{j} \ldots v_{s}$ is a prorperly colored path of order at least $s+1$, a contradiction to the maximality of $P_{s}$. If $j=i+4$, then we have $c\left(x v_{i+3}\right)=c\left(v_{i+2} v_{i+3}\right) \quad$ and $\quad c\left(y v_{i+1}\right)=c\left(v_{i+1} v_{i+2}\right), \quad$ otherwise, $v_{1} v_{2} \ldots v_{i+3} x P_{x y} y v_{i+4} \ldots v_{s}$ or $v_{1} v_{2} \ldots v_{i+1} y P_{y x} x v_{i+3} \ldots v_{s}$ is a properly colored path of order at least $s+2$, a contradiction to the maximality of $P_{s}$. It follows that $v_{1} \ldots v_{i+1} y P_{y x} x v_{i+3} \ldots v_{s}$ is a properly colored path of order at least $s+1$, a contradiction to the maximality of $P_{s}$. If $j=i+5$, by a similar argument of the former case, we have $c\left(x v_{i+3}\right)=c\left(y v_{i+2}\right)=c\left(v_{i+2} v_{i+3}\right) \quad$ and $v_{1} v_{2} \ldots v_{i+2} y P_{y x} x v_{i+3} \ldots v_{s}$ is a properly colored path of order at least $s+2$, a contradiction to the maximality of $P_{s}$. 
By Claim 3, for any six consecutive vertices $v_{i}, v_{i+1}, v_{i+2}, v_{i+3}, v_{i+4}, v_{i+5} \in V\left(P_{S}\right)$ and any vertex $u \in V(H)$, we have $\left|E_{G}\left(u,\left\{v_{i}, v_{i+1}, v_{i+2}, v_{i+3}, v_{i+4}, v_{i+5}\right\}\right)\right| \leq 2$. Thus, by Claim 5, we have

$$
\left|E_{G}\left(V(H),\left\{v_{i}, v_{i+1}, v_{i+2}, v_{i+3}, v_{i+4}, v_{i+5}\right\}\right)\right| \leq \max \{2,|V(H)|\} \leq|V(H)| .
$$

Combining (3.6) and (3.7), we have

$$
\left|E_{G}\left(V(H), V\left(P_{s}\right)\right)\right| \leq\left\lceil\frac{s-2(2 r+1)}{6}\right\rceil|V(H)| .
$$

Combining (3.5) and (3.8), we have

$$
\begin{aligned}
\left|E_{G}\left(V(H), V\left(P_{s}\right)\right)\right|+|E(H)| & \leq\left\lceil\frac{s-2(2 r+1)}{6}\right\rceil|V(H)|+\frac{r|V(H)|-r}{2} \\
& \leq\left(\left\lceil\frac{s-4 r-2}{6}\right\rceil+\frac{r}{2}\right)|V(H)| \\
& \leq\left(\left\lceil\frac{s-4 r-2}{6}+\frac{r+1}{2}\right\rceil\right)|V(H)| \\
& \leq\left\lceil\frac{s-1}{6}\right\rceil|V(H)|
\end{aligned}
$$

The last inequality holds since $r \geq 2$. Note that $\left|E_{G}\left(V(H), V\left(P_{s}\right)\right)\right|+$ $|E(H)| \leq\left[\frac{s-1}{6}\right]|V(H)|$ holds for each component $H$ of $G\left[U_{3}\right]$. Thus, we have

$$
\left|E_{G}\left(U_{3}, P_{s}\right)\right|+\left|E\left(G\left[U_{3}\right]\right)\right| \leq\left\lceil\frac{s-1}{6}\right\rceil\left|U_{3}\right| \leq\left\lceil\frac{l-2}{6}\right\rceil\left|U_{3}\right| .
$$

By (3.3), (3.4) and (3.9), we have

$$
\begin{aligned}
\operatorname{pr}\left(K_{n}, P_{l}\right) & =\left|C\left(K_{n}\right)\right| \leq\left|C\left(P_{s}\right)\right|+|E(G)| \\
& \leq\left(\begin{array}{c}
s \\
2
\end{array}\right)+\left|E\left(G\left[U_{1}\right]\right)\right|+\left|E_{G}\left(U_{2}, P_{s}\right)\right|+\left|E_{G}\left(U_{3}, P_{s}\right)\right|+\left|E\left(G\left[U_{3}\right]\right)\right| \\
& \leq\left(\begin{array}{l}
s \\
2
\end{array}\right)+\left(\frac{1}{2}\left\lfloor\frac{l-1}{2}\right\rfloor-1\right)\left|U_{1}\right|+\left(\left\lfloor\frac{l}{3}\right\rfloor-1\right)\left|U_{2}\right|+\left\lceil\frac{l-2}{6}\right\rceil\left|U_{3}\right| .
\end{aligned}
$$

Note that $\frac{1}{2}\left\lfloor\frac{l-1}{2}\right\rfloor-1 \leq\left\lfloor\frac{l}{3}\right\rfloor-1-\frac{1}{2}$ for $l \geq 6$ and $\left\lceil\frac{l-2}{6}\right\rceil \leq\left\lfloor\frac{l}{3}\right\rfloor-1-\frac{1}{2}$ for all $l \geq 12$. When $l \leq 11$, we have $s \leq 10$, by Claim $4, U_{3}=\emptyset$. Let $U^{*}=\left\{u \in U_{2}: d_{G}(u)=\right.$ $\left.\left\lfloor\frac{l}{3}\right\rfloor-1\right\}$. Then we have

$$
\operatorname{pr}\left(K_{n}, P_{l}\right) \leq\left(\begin{array}{l}
s \\
2
\end{array}\right)+\left(\left\lfloor\frac{l}{3}\right\rfloor-1-\frac{1}{2}\right)\left(n-s-\left|U^{*}\right|\right)+\left(\left\lfloor\frac{l}{3}\right\rfloor-1\right)\left|U^{*}\right| .
$$

Since $n \geq 2 l^{3}$, by Proposition 2, we have 


$$
\operatorname{pr}\left(K_{n}, P_{l}\right) \geq\left(\left\lfloor\frac{l}{3}\right\rfloor-1\right) n-\left(\begin{array}{c}
\left\lfloor\frac{l}{3}\right\rfloor \\
2
\end{array}\right)+1+r_{l}
$$

Combining (3.10) and (3.11), since $n \geq 2 l^{3}$, we have $\left|U^{*}\right| \geq l^{3}$. By Claims 1 and 3, there are at most $\left(\begin{array}{c}s-4-2\left(\left\lfloor\frac{l}{3}\right\rfloor-1-1\right) \\ \left\lfloor\frac{l}{3}\right\rfloor-1\end{array}\right)$ distinct $\left(\left\lfloor\frac{l}{3}\right\rfloor-1\right)$-subset of $V\left(P_{s}\right)$ can be the neighborhood of some vertex in $U^{*}$. Since $s \leq l-1$ and $6 \leq l \leq 3\left\lfloor\frac{l}{3}\right\rfloor+2$, we have

$$
\left(\begin{array}{c}
s-4-2\left(\left\lfloor\frac{l}{3}\right\rfloor-1-1\right) \\
\left\lfloor\frac{l}{3}\right\rfloor-1
\end{array}\right) \leq\left(\begin{array}{c}
l-1-2\left\lfloor\frac{l}{3}\right\rfloor \\
\left\lfloor\frac{l}{3}\right\rfloor-1
\end{array}\right) \leq\left(\begin{array}{c}
\left\lfloor\frac{l}{3}\right\rfloor+1 \\
\left\lfloor\frac{l}{3}\right\rfloor-1
\end{array}\right)=\left(\begin{array}{c}
\left\lfloor\frac{l}{3}\right\rfloor+1 \\
2
\end{array}\right) \leq \frac{l^{2}}{9} .
$$

Note that $\left|U^{*}\right| \geq l^{3}>\frac{l^{2}}{9}\left(2\left\lfloor\frac{l}{3}\right\rfloor+3\right)$, by Pigeonhole Principle, $U^{*}$ contains at least $2\left\lfloor\frac{l}{3}\right\rfloor+3$ vertices which have a common neighborhood of size $\left\lfloor\frac{l}{3}\right\rfloor-1$ in $G$. That is, we find a rainbow $K_{\left\lfloor\frac{l}{3}\right\rfloor-1,2\left\lfloor\frac{l}{3}\right\rfloor+3}$. By Proposition 3 , the proof is complete.

\section{Cycles}

The lower bound of $\operatorname{pr}\left(K_{n}, C_{k}\right)$ was given roughly by Manoussakis, Spyratos, Tuza and Voigt in [15]. Here we prove the lower bound precisely again.

Proposition 4 Let $C_{k}$ be a cycle on $k$ vertices and $(k-1) \equiv r_{k-1}(\bmod 3)$, where $0 \leq r_{k-1} \leq 2$. For $n \geq k$,

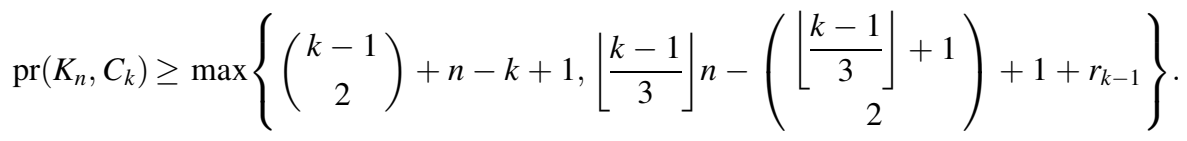

Proof We color the edges of $K_{n}$ as follows. For the first lower bound, we choose a $K_{k-1}$ and color it rainbow, and use one extra color for all the remaining edges. In such way, we use exactly $\left(\begin{array}{c}k-1 \\ 2\end{array}\right)+1$ colors and do not obtain a properly colored $C_{k}$.

For the second lower bound, we partition $K_{n}$ into two graphs $K_{\left\lfloor\frac{k-1}{3}\right\rfloor}+\bar{K}_{n-\left\lfloor\frac{k-1}{3}\right\rfloor}$ and $K_{n-\left\lfloor\frac{k-1}{3}\right\rfloor}$. First we color $K_{\left\lfloor\frac{k-1}{3}\right\rfloor}+\bar{K}_{n-\left\lfloor\frac{k-1}{3}\right\rfloor}$ rainbow. Then we color $K_{n-\left\lfloor\frac{k-1}{3}\right\rfloor}$ by $(1+$ $\left.r_{k-1}\right)$ new colors without producing a properly colored $P_{3+r_{k-1}}$ (See the proof of 
Proposition 3.1). In such way, we use exactly $\left\lfloor\frac{k-1}{3}\right\rfloor n-\left(\left\lfloor\frac{k-1}{3}\right\rfloor+1\right)+1+r_{k-1}$ colors and do not obtain a properly colored $C_{k}$.

Conjecture 3 Let $C_{k}$ be a cycle on $k$ vertices and $(k-1) \equiv r_{k-1}(\bmod 3)$, where $0 \leq r_{k-1} \leq 2$. For $n \geq k$,

$$
\operatorname{pr}\left(K_{n}, C_{k}\right)=\max \left\{\left(\begin{array}{c}
k-1 \\
2
\end{array}\right)+n-k+1,\left\lfloor\frac{k-1}{3}\right\rfloor n-\left(\left\lfloor\frac{k-1}{3}\right\rfloor+1\right)+1+r_{k-1}\right\} .
$$

Now we prove Conjecture 2 holds for $C_{5}$ and $C_{6}$, respectively.

Theorem 4 For $n \geq 5, \operatorname{pr}\left(K_{n}, C_{5}\right)=n+2$.

Proof By Proposition 4, we have $\operatorname{pr}\left(K_{n}, C_{5}\right) \geq n+2$ for $n \geq 5$. We will prove $\operatorname{pr}\left(K_{n}, C_{5}\right) \leq n+2$ by induction on $n$. The base cases $n=5$ and $n=6$ follow from (1.3) and (1.4), respectively. For $n \geq 7$, assume that the conclusion holds for order less than $n$. Let $c$ be an $(n+3)$-edge-coloring of $K_{n}$. If there is a vertex $v$ such that $d^{c}(v) \leq 1$, then $\left|C\left(K_{n}-v\right)\right| \geq n+3-1=(n-1)+3$ and there is a properly colored $C_{5}$ by the induction hypothesis. Thus we assume that $d^{c}(v) \geq 2$, for all $v \in V\left(K_{n}\right)$. Let $G$ be the weak representing subgraph of $K_{n}$. By (1.5), we have $|E(G)| \geq 2 n-(n+3)=n-3 \geq 4$. Thus, $G$ contains a 2-matching. Let $\{x y, z w\}$ be a 2-matching of $G$. Choose a vertex $u \in V\left(K_{n}\right) \backslash\{x, y, z, w\}$. We consider the following two cases.

Case 1. There are at least two edges of $\{u x, u y, u z, u w\}$ are colored with distinct colors.

In this case, there are at least one edge of $\{u x, u y\}$, we say $u x$, and at least one edge of $\{u z, u w\}$, we say $u z$, such that $c(u x) \neq c(u z)$. By the definition of $G$, we have $c(u x) \neq c(x y), c(u z) \neq c(z w)$ and $c(x y) \neq c(y w) \neq c(z w)$. Thus, uxywzu is a properly colored $C_{5}$.

Case 2. The four edges $u x, u y, u z$ and $u w$ are colored with the same color.

If $c(u x)$ is starred at $u$, since $d^{c}(u) \geq 2$, there exists a vertex $v \in$ $V\left(K_{n}\right) \backslash\{x, y, z, w, u\}$ such that $c(u v)$ is starred at $u$ and $c(u v) \neq c(u x)$. Also, we have $c(u x) \neq c(x z) \neq c(z w)$ and $c(z w) \neq c(v w) \neq c(u v)$. Thus, uxzwvu is a properly colored $C_{5}$. If $c(u x)$ is not starred at $u$, since $d^{c}(u) \geq 2$, there exists two vertices $v_{1}, v_{2} \in V\left(K_{n}\right) \backslash\{x, y, z, w, u\}$ such that $c\left(u v_{1}\right)$ and $c\left(u v_{2}\right)$ are starred at $u$ and $c\left(u v_{1}\right) \neq c\left(u v_{2}\right)$. Also, we have $c\left(u v_{1}\right) \neq c\left(v_{1} x\right) \neq c(x y)$ and $c\left(u v_{2}\right) \neq c\left(v_{2} z\right) \neq c(x y)$. Thus, $u v_{1} x y v_{2} u$ is a properly colored $C_{5}$.

For $C_{6}$, we consider more cases to prove it.

Theorem 5 For $n \geq 6, \operatorname{pr}\left(K_{n}, C_{6}\right)=n+5$. 
Proof By Proposition 4, we have $\operatorname{pr}\left(K_{n}, C_{6}\right) \geq n+5$ for $n \geq 6$. We will prove $\operatorname{pr}\left(K_{n}, C_{6}\right) \leq n+5$ by induction on $n$. The base cases $n=6$ and $n=7$ follow from (1.3) and (1.4), respectively. For $n \geq 8$, assume that the conclusion holds for order less than $n$. Let $c$ be an $(n+6)$-edge-coloring of $K_{n}$. If there is a vertex $v$ such that $d^{c}(v) \leq 1$, then $\left|C\left(K_{n}-v\right)\right| \geq n+6-1=(n-1)+6$ and there is a properly colored $C_{6}$ by the induction hypothesis. Thus we assume that $d^{c}(v) \geq 2$ for all $v \in V\left(K_{n}\right)$. Let $G$ be the weak representing subgraph of $K_{n}$. By (1.5), we have $|E(G)| \geq 2 n-(n+6)=n-6 \geq 2$.

Case 1. $\Delta(G) \geq 2$.

In this case, $G$ contains a path of order 3. Let $P_{3}=x y z$ be such a path of $G$ and $U=V\left(K_{n}\right) \backslash\{x, y, z\}$. Let $H$ be a subgraph $K_{n}$ obtained by choosing one edge from the colors which are starred at some vertex of $U$ such that the number of edges between $\{x, y, z\}$ and $U$ is as large as possible.

Case $1.1|E(H[U])| \geq 2$.

Let $u_{1} u_{2}, v_{1} v_{2} \in E(H[U])$. If $u_{1} u_{2}$ and $v_{1} v_{2}$ have a common end vertex, we say $u_{2}=v_{1}$, then $c\left(x u_{1}\right) \neq c\left(u_{1} v_{1}\right)$ and $c\left(z v_{2}\right) \neq c\left(v_{1} v_{2}\right)$ by the choice of $H$. Thus $x y z v_{2} v_{1} u_{1} x$ is a properly colored $C_{6}$. Now we may assume that $\left\{u_{1} u_{2}, v_{1} v_{2}\right\}$ is a 2matching of $H$. Assume that $c\left(u_{1} u_{2}\right)$ and $c\left(v_{1} v_{2}\right)$ are starred at $u_{1}$ and $v_{1}$ respectively. Thus $c\left(u_{2} v_{2}\right) \neq c\left(u_{1} u_{2}\right)$ and $c\left(u_{2} v_{2}\right) \neq c\left(v_{1} v_{2}\right)$. By the choice of $H$, we have $c\left(x u_{1}\right) \neq c\left(u_{1} u_{2}\right)$ and $c\left(y v_{1}\right) \neq c\left(v_{1} v_{2}\right)$. Thus, $x y v_{1} v_{2} u_{2} u_{1} x$ is a properly colored $C_{6}$.

Case $1.2|E(H[U])|=1$.

Assume $u v \in E(H[U])$ and $c(u v)$ is starred at $u$. Then we have $c(x u) \neq c(u v)$. Also, $c(v z) \neq c(u v)$. Take a vertex $w \in U \backslash\{u, v\}$. Since $d^{c}(w) \geq 2$, we have $\left|E_{H}(w,\{x, y, z\})\right| \geq 2$. There is at least one of $\{x, z\}$, say $x$, such that $c(w x)$ is starred at $w$ and $c(w x) \neq c(w y)$. Also, we have $c(w x) \neq c(u x)$. Thus wxuvzyw is a properly colored $C_{6}$.

Case 1.3 $E(H[U])=\emptyset$.

For all $v \in U$, since $d^{c}(v) \geq 2$, we have $\left|E_{H}(v,\{x, y, z\})\right| \geq 2$. Notice that $|U| \geq n-3 \geq 5$. If there are three vertices in $U$, say $u_{1}, u_{2}, u_{3} \in U$, such that they have a common neighborhood $\{x, z\}$ in $H$, then at least one of $\left\{u_{1} x, u_{1} z\right\}$, say $u_{1} x$, such that $c\left(u_{1} y\right) \neq c\left(u_{1} x\right)$. Also, at most one edge of $\left\{u_{2} x, u_{2} z, u_{3} x, u_{3} z\right\}$ has the same color as $c\left(u_{2} u_{3}\right)$. Thus, at least one of $x u_{1} y z u_{3} u_{2} x$ and $x u_{1} y z u_{2} u_{3} x$ is a properly colored $C_{6}$.

Now we may assume that there are at least two vertices in $U$, say $u_{1}, u_{2}$, such that they have a common neighborhood $\{x, y\}$ or $\{y, z\}$ in $H$, say $\{x, y\}$. If there is a vertex $u_{3} \in U \backslash\left\{u_{1}, u_{2}\right\} \quad$ such that $u_{3} y, u_{3} z \in E(H)$, we have $c(z x) \notin$ $\left\{c\left(x u_{1}\right), c\left(x u_{2}\right), c\left(z u_{3}\right)\right\}$ and at most one edge of $\left\{u_{1} x, u_{1} y, u_{2} x, u_{2} y\right\}$ has the same color as $c\left(u_{1} u_{2}\right)$. Thus, at least one of $x u_{1} u_{2} y u_{3} z x$ and $x u_{2} u_{1} y u_{3} z x$ is a properly colored $C_{6}$. If there is a vertex $u_{3} \in U \backslash\left\{u_{1}, u_{2}\right\}$ such that $u_{3} x, u_{3} z \in E(H)$, at least one of $x u_{1} u_{2} y z u_{3} x$ and $x u_{2} u_{1} y z u_{3} x$ is a properly colored $C_{6}$. Now we may assume that $U$ has a common neighborhood $\{x, y\}$ in $H$. Take four distinct vertices $u_{1}, u_{2}, u_{3}, u_{4} \in U$. At most one edge of $\left\{u_{1} x, u_{1} y, u_{2} x, u_{2} y\right\}$ has the same color as $c\left(u_{1} u_{2}\right)$ and at most one edge of $\left\{u_{3} x, u_{3} y, u_{4} x, u_{4} y\right\}$ has the same color as $c\left(u_{3} u_{4}\right)$. Thus the graph induced by the edges set $\left\{u_{1} u_{2}, u_{3} u_{4}, x u_{i}, y u_{i}: 1 \leq i \leq 4\right\}$ contains a 
properly colored $C_{6}$.

Case 2. $\Delta(G)=1$.

Note that if $G$ has three independent edges, then we can find a properly colored $C_{6}$. Recall that $|E(G)| \geq n-6 \geq 2$. Now we may assume that $n=8$ and $|E(G)|=2$. Let $E(G)=\{x y, z w\}$ and $U=V\left(K_{8}\right) \backslash\{x, y, z, w\}=\left\{u_{1}, u_{2}, u_{3}, u_{4}\right\}$.

Case 2.1 There is an edge $u_{i} u_{j}$ such that $c\left(u_{i} u_{j}\right)$ is starred at $u_{i}$, say $c\left(u_{1} u_{2}\right)$ is starred at $u_{1}$.

If there is one vertex in $\{x, y, z, w\}$, say $x$, such that $c\left(u_{1} x\right) \neq c\left(u_{1} u_{2}\right)$, then $u_{1} x y z w u_{2} u_{1}$ is a properly colored $C_{6}$. We assume that $c\left(u_{1} x\right)=c\left(u_{1} y\right)=c\left(u_{1} z\right)=$ $c\left(u_{1} w\right)=c\left(u_{1} u_{2}\right)$. Since $d^{c}\left(u_{1}\right) \geq 2$, we can assume that $c\left(u_{1} u_{3}\right)$ is starred at $u_{1}$ and $c\left(u_{1} u_{3}\right) \neq c\left(u_{1} u_{2}\right)$. Thus $u_{1} x y z w u_{3} u_{1}$ is a properly colored $C_{6}$.

Case 2.2 For all edge $u_{i} u_{j}, c\left(u_{i} u_{j}\right)$ is not starred at $u_{i}$ or $u_{j}$.

Since $d^{c}\left(u_{1}\right) \geq 2$ and $d^{c}\left(u_{2}\right) \geq 2$, we can find two distinct vertices $v_{1}, v_{2} \in$ $\{x, y, z, w\}$ such that $c\left(u_{1} v_{1}\right)$ is starred at $u_{1}$ and $c\left(u_{2} v_{2}\right)$ is starred at $u_{2}$. If $v_{1}=x$ and $v_{2}=y$, then $u_{1} x z w y u_{2} u_{1}$ is a properly colored $C_{6}$. If $v_{1}=x$ and $v_{2}=z$, then $u_{1} x y w z u_{2} u_{1}$ is a properly colored $C_{6}$.

\section{$5 K_{4}^{-}$and $K_{2,3}$}

In this section, we will prove Theorems 6 and 7. First, we determine the exact value of $\operatorname{pr}\left(K_{n}, K_{4}^{-}\right)$.

Theorem 6 For $n \geq 4, \operatorname{pr}\left(K_{n}, K_{4}^{-}\right)=\left\lfloor\frac{3(n-1)}{2}\right\rfloor$.

Proof The lower bound: Consider an edge-coloring of $K_{n}$ as follows. Take a triangle $C_{3}=x y z$ of $K_{n}$ and a maximum matching $M=\left\{x_{1} y_{1}, x_{2} y_{2}, \ldots, x_{\left\lfloor\frac{n-3}{2}\right\rfloor} y_{\left\lfloor\frac{n-3}{2}\right\rfloor}\right\}$ of $K_{n}-\{x, y, z\}$. There is one vertex $w$ in $V\left(K_{n}\right) \backslash(V(M) \cup\{x, y, z\})$ when $n$ is even. For $\quad 1 \leq i \leq\left\lfloor\frac{n-3}{2}\right\rfloor, \quad$ color all the edges of $\left\{u x_{i}: u \in\right.$ $\left.\left\{x, y, z, x_{1}, y_{1}, x_{2}, y_{2}, \ldots, x_{i-1}, y_{i-1}\right\}\right\}$ with color $c_{1 i}$ and all the edges of $\left\{u y_{i}: u \in\right.$ $\left.\left\{x, y, z, x_{1}, y_{1}, x_{2}, y_{2}, \ldots, x_{i-1}, y_{i-1}\right\}\right\}$ with color $c_{2 i}$. If $n$ is even, color all edges of $\left\{u w: u \in V\left(K_{n}-w\right)\right\}$ with a new color. Finally, assign distinct new colors to all edges of $C_{3} \cup M$. In such a coloring, there is no properly colored $K_{4}^{-}$, and the number of colors is $\left\lfloor\frac{3(n-1)}{2}\right\rfloor$.

The upper bound: We will prove that for any $\left\lfloor\frac{3 n-1}{2}\right\rfloor$-edge-coloring of $K_{n}$, there is a properly colored $K_{4}^{-}$by induction on $n$. The base case $n=4$ is trivial. For $n \geq 5$, assume that the conclusion holds for order less than $n$. Let $c$ be a $\left\lfloor\frac{3 n-1}{2}\right\rfloor$-edgecoloring of $K_{n}$. If there is a vertex $v$ such that $d^{c}(v) \leq 1$, then $\left|C\left(K_{n}-v\right)\right| \geq\left\lfloor\frac{3 n-1}{2}\right\rfloor-1 \geq\left\lfloor\frac{3(n-1)-1}{2}\right\rfloor$, and there is a properly colored $K_{4}^{-}$in $K_{n}-$ $v$ by the induction hypothesis. We may assume that $d^{c}(v) \geq 2$ for all $v \in V\left(K_{n}\right)$. Let $G$ be the weak representing subgraph of $K_{n}$. By (1.5), we have $|E(G)| \geq 2 n-\left\lfloor\frac{3 n-1}{2}\right\rfloor=\left\lceil\frac{n+1}{2}\right\rceil$, which implies there is a path $P_{3}=x y z$ in $G$. By the construction of $G$, if $e=u v \in E(G)$, the $c(e)$ is starred at $u$ and $v$. We consider the following two cases. 
Case 1. $x z \notin E(G)$.

In this case, $c(x z)$ is not starred at $x$ or $z$, say $x$. Since $d^{c}(x) \geq 2$, there is a vertex $w \notin\{x, y, z\}$ such that $c(x w)$ is starred at $x$. Then $c(x z), c(y w) \notin$ $\{c(x y), c(y z), c(x w)\}$ and the edge set $\{x y, y z, x z, x w, y w\}$ induces a properly colored $K_{4}^{-}$.

Case 2. $x z \in E(G)$.

In this case, we can assume $c(u x)=c(u y)=c(u z)$ for all $u \in V\left(K_{n}\right) \backslash\{x, y, z\}$; otherwise we easily have a properly colored copy of $K_{4}^{-}$in $K_{n}[x, y, z, u]$. Thus we have

$$
\left|C\left(K_{n}-\{x, y\}\right)\right| \geq\left\lfloor\frac{3 n-1}{2}\right\rfloor-3=\left\lfloor\frac{3(n-2)-1}{2}\right\rfloor .
$$

If $n=5$, then $3=\left|E\left(K_{5}-\{x, y\}\right)\right| \geq\left|C\left(K_{5}-\{x, y\}\right)\right| \geq 4$, a contradiction. Thus we may assume that $n \geq 6$, there is a properly colored $K_{4}^{-}$in $K_{n}-\{x, y\}$ by the induction hypothesis.

Now we prove the lower bound and upper bound of $\operatorname{pr}\left(K_{n}, K_{2,3}\right)$. We conjecture that the exact value is closer to the lower bound.

Theorem 7 For $n \geq 5, \frac{7}{4} n+O(1) \leq \operatorname{pr}\left(K_{n}, K_{2,3}\right) \leq 2 n-1$.

Proof The lower bound: Let $n=4 k+r$, where $1 \leq r \leq 4$. Set $V\left(K_{n}\right)=V_{1} \cup \cdots \cup$ $V_{k} \cup V_{k+1}$ such that $V_{i} \cap V_{j}=\emptyset$ for $i \neq j,\left|V_{i}\right|=4$ for $1 \leq i \leq k$ and $\left|V_{k+1}\right|=r$. We color the edges with end-vertices in the same set with $6 k+\left(\begin{array}{l}r \\ 2\end{array}\right)$ distinct colors and color the remaining edges with $k$ addition colors $c_{1}, c_{2}, \ldots, c_{k}$ such that all edges between $V_{i}$ and $V_{j}$ are colored with $c_{\min \{i, j\}}$, where $i \neq j$. The total number of colors is $\frac{7}{4} n+O(1)$ and there is no properly colored $K_{2,3}$.

The upper bound: We will prove that for any $2 n$ edge-coloring of $K_{n}$, there is a properly colored $K_{2,3}$ by induction on $n$. The base case $n=5$ is trivial. For $n \geq 6$, assume that the conclusion holds for order less than $n$. Let $c$ be a $2 n$-edge-coloring of $K_{n}$. If there is a vertex $v$ such that $d^{c}(v) \leq 2$, then $\left|C\left(K_{n}-v\right)\right| \geq 2 n-2$ and there is a properly colored $K_{2,3}$ in $K_{n}-v$ by the induction hypothesis. We may assume that $d^{c}(v) \geq 3$ for all $v \in V\left(K_{n}\right)$. Let $G$ be the weak representing subgraph of $K_{n}$. By (1.5), we have $|E(G)| \geq 3 n-2 n=n$. Note that for $n \geq 4, \operatorname{ex}\left(n, P_{4}\right) \leq n$ and the equality holds for the graph of disjoint copies of $C_{3}$ (see [5]). So we will consider the following two cases.

Case 1. $G$ contains a $P_{4}=x y z w$.

If $G\left[V\left(P_{4}\right)\right] \cong K_{4}$, then we can assume $c(u x)=c(u y)=c(u z)=c(u w)$ for all $u \in V\left(K_{n}\right) \backslash\{x, y, z, w\}$; otherwise we easily have a properly colored copy of $K_{2,3}$. Therefore

$$
\left|C\left(K_{n}-\{x, y, z\}\right)\right| \geq 2 n-6=2(n-3) .
$$

If $n=6$, then $3=\left|E\left(K_{6}-\{x, y, z\}\right)\right| \geq\left|C\left(K_{6}-\{x, y, z\}\right)\right| \geq 6$, a contradiction. If $n=7$, then $6=\left|E\left(K_{6}-\{x, y, z\}\right)\right| \geq\left|C\left(K_{6}-\{x, y, z\}\right)\right| \geq 8$, a contradiction. Thus we may assume that $n \geq 8$, there is a properly colored $K_{2,3}$ in $K_{n}-\{x, y, z\}$ by the 
induction hypothesis.

Now we consider the case $G\left[V\left(P_{4}\right)\right] \not K_{4}$. Since $d^{c}(x) \geq 3$ and $d^{c}(w) \geq 3$, there is a vertex $u \in V\left(K_{n}\right) \backslash\{x, y, z, w\}$ such that $c(x u)$ or $c(w u)$, say $c(x u)$ is starred at $x$ and $c(x u) \notin\{c(x y), c(x w)\}$. Therefore, the edges between $\{x, z\}$ and $\{y, u, w\}$ induce a properly colored $K_{2,3}$.

Case 2. $G$ is the graph of disjoint copies of $C_{3}$.

Let $T_{1}=x y z x$ be a triangle of $G$. Since $d^{c}(x) \geq 3$, there is a vertex $u \in$ $V\left(K_{n}\right) \backslash\{x, y, z\}$ such that $c(x u)$ is starred at $x$ and $c(x u) \notin\{c(x y), c(x z)\}$. Suppose $u$ belong to the triangle $T_{2}=u v w u$ of $G$. Therefore, the edges between $\{y, u\}$ and $\{x, z, v\}$ induce a properly colored $K_{2,3}$.

\section{Conclusion}

In this paper, we obtain the relationship of $\operatorname{pr}\left(K_{n}, G\right)$ and $\operatorname{ex}\left(n, \mathcal{G}^{\prime}\right)$ by Theorem 2 . We also determine the value of $\operatorname{pr}\left(K_{n}, G\right)$ for some small graphs. Since the lower bound of $\operatorname{pr}\left(K_{n}, C_{k}\right)$ is very similar to the paths, we expect that the idea of the proof of Theorem 3 would be helpful to prove Conjecture 2 for large $n$.

Another interesting open problem is determining the behavior of $\operatorname{pr}\left(K_{n}, K_{4}\right)$. Theorem 1 shows that $\operatorname{pr}\left(K_{n}, K_{4}\right)=o\left(n^{2}\right)$ and Theorem 2 shows that $\operatorname{pr}\left(K_{n}, K_{4}\right) \geq \operatorname{ex}\left(n, C_{4}\right)+1$. Since $\operatorname{ex}\left(n, C_{4}\right)=\frac{1}{2} n^{3 / 2}+o\left(n^{3 / 2}\right)$ (See [4, 6]), one can prove that $\operatorname{pr}\left(K_{n}, K_{4}\right)=O\left(n^{3 / 2}\right)$. The main idea is that for an edge-coloring of $K_{n}$, if the weak representing subgraph contains a $C_{4}$, then there exists a properly colored $K_{4}$ in $K_{n}$.

Acknowledgements The authors are thankful to the anonymous referee for his/her useful comments.

Funding Open access funding provided by ELKH Alfréd Rényi Institute of Mathematics.

Open Access This article is licensed under a Creative Commons Attribution 4.0 International License, which permits use, sharing, adaptation, distribution and reproduction in any medium or format, as long as you give appropriate credit to the original author(s) and the source, provide a link to the Creative Commons licence, and indicate if changes were made. The images or other third party material in this article are included in the article's Creative Commons licence, unless indicated otherwise in a credit line to the material. If material is not included in the article's Creative Commons licence and your intended use is not permitted by statutory regulation or exceeds the permitted use, you will need to obtain permission directly from the copyright holder. To view a copy of this licence, visit http:// creativecommons.org/licenses/by/4.0/.

\section{References}

1. Alon, N.: On a conjecture of Erdős, Simonovits and Sós concerning anti-Ramsey theorems. J. Graph Theory 7(1), 91-94 (1983)

2. Axenovich, M., Jiang, T.: Anti-Ramsey numbers for small complete bipartite graphs. Ars Combin. 73, 311-318 (2004) 
3. Bialostocki, A., Gilboa, S., Roditty, Y.: Anti-Ramsey number of small graphs. Ars Combin. 123, 41-53 (2015)

4. Brown, W.G.: On graphs that do not contain a Thomsen graph. Can. Math. Bull. 9, 281-285 (1966)

5. Erdös, P., Gallai, T.: On maximal paths and circuits of graphs. Acta Math. Acad. Sci. Hung. 10, 337-356 (1959)

6. Erdős, P., Rényi, A., Sós, V.T.: On a problem of graph theory. Stud. Sci. Math. Hung. 1, 215-235 (1966)

7. Erdős, P., Simonovits, M.: A limit theorem in graph theory. Stud. Sci. Math. Hung. 1, 51-57 (1996)

8. Erdős, P., Simonovits, M., Sós, V.T.: Anti-Ramsey theorems. In Infinite and Finite Sets (Colloq. Keszthely 1973). Colloq. Math. Soc. János Bolyai 10, 633-643 (1975)

9. Erdős, P., Stone, A.H.: On the structure of linear graphs. Bull. Am. Math. Soc. 52, 1087-1091 (1946)

10. Fujita, S., Magnant, C., Ozeki, K.: Rainbow generalizations of Ramsey theory: a survey. Graphs Combin. 26(1), 1-30 (2010)

11. Jiang, T.: Edge-coloring with no large polychromatic stars. Graphs Combin. 18(2), 303-308 (2002)

12. Jiang, T., West, D.B.: Edge colorings of complete graphs that avoid polychromatic trees. Discrete Math. 274(1-3), 137-147 (2004)

13. Krop, E., York, M.: On anti-Ramsey numbers for complete bipartite graphs and the Turán function. arXiv:1108.5204

14. Li, R., Broersma, H., Zhang, S.: Properly edge-colored theta graphs in edge-colored complete graphs. Graphs Combin. 35(1), 261-286 (2019)

15. Manoussakis, Y., Spyratos, M., Tuza, Z.S., Voigt, M.: Minimal colorings for properly colored subgraphs. Graphs Combin. 12(4), 345-360 (1996)

16. Montellano-Ballesteros, J.J.: An anti-Ramsey theorem on diamonds. Graphs Combin. 26(2), 283-291 (2010)

17. Montellano-Ballesteros, J.J., Neumann-Lara, V.: An anti-Ramsey theorem. Combinatorica 22(3), 445-449 (2002)

18. Montellano-Ballesteros, J.J., Neumann-Lara, V.: An anti-Ramsey theorem on cycles. Graphs Combin. 21(3), 343-354 (2005)

19. Schiermeyer, I.: Rainbow numbers for matchings and complete graphs. Discrete Math. 286(1-2), 157-162 (2004)

20. Simonovits, M., Sós, V.T.: On restricted colorings of $K_{n}$. Combinatorica 4(1), 101-110 (1984)

21. Xu, C., Magnant, C., Zhang, S.: Properly colored $C_{4}$ 's in edge-colored graphs. Discrete Math. 343(12), 112116 (2020)

Publisher's Note Springer Nature remains neutral with regard to jurisdictional claims in published maps and institutional affiliations. 